\title{
High-Density Lipoprotein-Targeted Therapy and Apolipoprotein A-I Mimetic Peptides
}

\author{
Yoshinari Uehara, MD, PhD; Giulia Chiesa, PhD; Keijiro Saku, MD, PhD
}

\begin{abstract}
Numerous randomized clinical trials have established statins as the major standard therapy for atherosclerotic diseases because these molecules decrease the plasma level of low-density lipoprotein (LDL) cholesterol and moderately increase that of plasma high-density lipoprotein (HDL) cholesterol. The reverse cholesterol transport pathway, mediated by HDL particles, has a relevant antiatherogenic potential. An important approach to HDL-targeted therapy is optimization of the HDL-cholesterol level and enhanced removal of plasma cholesterol, together with the prevention and mitigation of inflammation related to atherosclerosis. Small-molecule inhibitors of cholesteryl ester transfer protein (CETP) increase the HDL-cholesterol level in subjects with normal or low HDL-cholesterol. However, CETP inhibitors do not seem to reduce the risk of atherosclerotic diseases. HDL therapies using reconstituted HDL, including apolipoprotein (Apo) A-I Milano, ApoA-I mimetics, or full-length ApoA-I, are dramatically effective in animal models. Of those, the ApoA-I-mimetic peptide called FAMP effectively removes cholesterol via the ABCA1 transporter and acts as an antiatherosclerotic agent by enhancing the biological functions of HDL without elevating the HDL-cholesterol level. Our review of the literature leads us to conclude that HDL-targeted therapies have significant atheroprotective potential and thus may effectively treat patients with cardiovascular diseases. (Circ J 2015; 79: 2523-2528)
\end{abstract}

Key Words: Apolipoprotein; Atherosclerosis; Cardiovascular disease; Dyslipidemia; Reverse cholesterol transport

D yslipidemia is one of the more relevant risk factors for coronary artery disease. Previous epidemiological studies and clinical trials demonstrated an inverse association between the serum level of high-density lipoprotein (HDL) cholesterol and the risk of cardiovascular events and that HDL-cholesterol exerts many potential antiatherogenic effects. ${ }^{1}$ For example, HDL particles transport cholesterol from peripheral cells to the liver and steroidogenic organs that use cholesterol for the synthesis of lipoproteins, bile acids, and steroid hormones. ${ }^{1}$ Low-density lipoprotein (LDL) cholesterol-lowering therapy moderately increases the plasma level of HDL cholesterol ${ }^{2-5}$ and intensive statin therapy reduces the volume of coronary plaque in patients with acute coronary syndrome (ACS). These results were not predicted by any of the indices in the patients' baseline lipid profiles or by the LDL-cholesterol levels at follow-up, but they did correlate with the LDL-/HDL-cholesterol ratio at follow-up. ${ }^{6} \mathrm{HDL}$ plays a major role in reverse cholesterol transport (RCT), and the RCT system has various potential antiatherogenic properties., ${ }^{1,2}$

\section{HDL Metabolism and RCT}

HDL is isolated using ultracentrifugation at a density range of $1.063-1.21 \mathrm{~g} / \mathrm{ml}$ (HDL2, $1.063-1.125 \mathrm{~g} / \mathrm{ml}$; HDL3, $1.125-$ $1.21 \mathrm{~g} / \mathrm{ml}) .{ }^{7}$ However, HDL comprises a heterogeneous group of particles that differ in size, density, composition of lipids and apolipoproteins (Apo), and electrophoretic mobility. Gel electrophoresis separates HDL into a major subfraction that identically migrates as alpha HDL and other subfractions with mobilities similar to that of pre- $\beta$ HDL. Alpha HDL is the most abundant HDL particle in human plasma, and pre- $\beta$ HDL represents only $2-14 \%$ of the ApoA-I population. ${ }^{8,9}$

HDL metabolism is associated with several HDL-related genes, and its synthesis involves a complex pathway. Although the underlying genetic defects in many patients with primary low-HDL cholesterolemia are not clearly understood, defects in 3 pivotal molecules are associated with low plasma HDLcholesterol levels: ApoA-I, lecithin cholesterol acyltransferase, and ATP-binding cassette (ABC) A1. ${ }^{10} \mathrm{HDL}$ metabolism involves at least 3 important steps. First, lipid-free or lipidpoor ApoA-I removes free cholesterol from peripheral cells via ABCA1 to form nascent HDL. Second, the nascent HDL transported by ABCA1 is lipidated to generate mature HDL. Third, the mature HDL interacts with other ApoB-containing lipoproteins, such as VLDL, IDL, and LDL. ABCA1 is a key molecule for generating HDL particles de novo, which is the

Received September 7, 2015; revised manuscript received October 15, 2015; accepted October 28, 2015; released online November 6, 2015

Department of Cardiology (Y.U., K.S.), Center for Preventive, Anti-aging and Regenerative Medicine (Y.U.), Fukuoka University Hospital, Fukuoka; Faculty of Sports and Health Science (Y.U.), Department of Molecular Cardiovascular Therapeutics (Y.U., K.S.), Department of Advenced Therapeutics for Cardiovascular Disease (K.S.), Fukuoka University, Fukuoka, Japan; and Department of Pharmacological and Biomolecular Sciences, Università degli Studi di Milano, Milano (G.C.), Italy

Mailing address: Yoshinari Uehara, MD, PhD or Keijiro Saku, MD, PhD, Department of Cardiology, Fukuoka University Hospital, 7-45-1

Nanakuma, Jonan-ku, Fukuoka 814-0180, Japan. E-mail: ueharay@fukuoka-u.ac.jp or saku-k@fukuoka-u.ac.jp

ISSN-1346-9843 doi:10.1253/circj.CJ-15-0960

All rights are reserved to the Japanese Circulation Society. For permissions, please e-mail: cj@j-circ.or.jp 


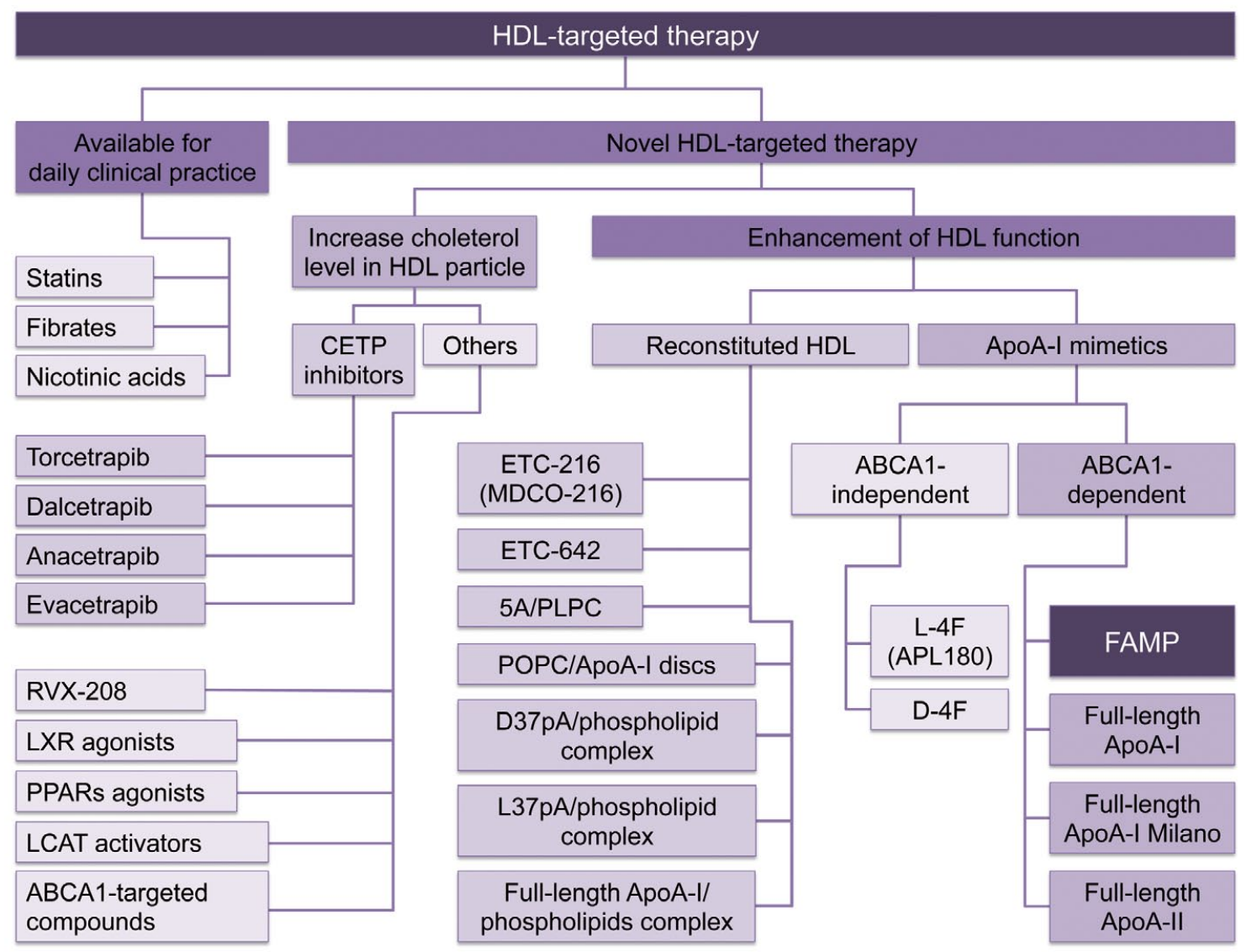

Figure 1. Several approaches to HDL-targeted therapy. ABCA1, ATP-binding cassette A1; Apo, apolipoprotein; CETP, cholesteryl ester transfer protein; FAMP, Fukuoka University APOA-I mimetic peptide; HDL, high-density lipoprotein; LCAT, lecithin cholesterol acyltransferase; LXR, liver $X$ receptor; PLPC, 1-palmitoyl-2-linoleoyl- sn-glycero-3-phosphocholine; POPC, 1-palmitoyl2-oleoylphosphatidylcholine; PPAR, peroxisome proliferator activated receptor.

initial step of RCT, because ABCA1 proteins transport cholesterol and phospholipids from the inner to the outer leaflet of the membrane. Lipid-poor ApoA-I subsequently removes these transported cholesterol and phospholipid molecules to form nascent HDL. ${ }^{11}$

\section{HDL-Targeted Therapy for Atherosclerosis}

\section{Inhibition of Cholesteryl Ester Transfer Protein (CETP)}

CETP regulates the cholesterol level of the HDL particles and mediates the transfer of the cholesteryl esters of HDL to ApoB-containing lipoproteins, as well as those of triglycerides from triglyceride-enriched lipoproteins to HDL. ${ }^{12}$ Patients with mutations in the gene encoding CETP produce high levels of HDL-cholesterol. ${ }^{13}$ Inhibitors of CETP activity, such as torcetrapib, dalcetrapib, anacetrapib, and evacetrapib, benefit subjects with normal or low levels of HDL-cholesterol by increasing its level. ${ }^{14,15}$ Furthermore, CETP deficiency markedly reduces the rate of ApoA-I turnover, accounting for the high HDL-cholesterol levels in humans with inherited hypercholesterolemia. ${ }^{16-18}$ Moreover, inhibition of CETP activity in subjects with low HDL cholesterol levels increases the cholesterol percentage in the HDL2 particle compared with that in the HDL 3 particle and increases the cholesterol levels of large
HDL particles. ${ }^{15}$ Therefore, inhibiting CETP activity is a pharmacological approach to raising the HDL-cholesterol level and potentially reducing the risk of cardiovascular disease. Small-molecule CETP inhibitors such as torcetrapib, ${ }^{19-22}$ dalcetrapib, ${ }^{23-25}$ anacetrapib, ${ }^{26,27}$ and evacetrapib, ${ }^{28,29}$ significantly increase HDL-cholesterol levels in humans. However, this pharmacological approach does not seem to be beneficial for atherosclerotic disease. For example, the ILLUSTRATE study failed to show any slowing in atherosclerosis progression and determined a significant increase in the number of deaths from cardiovascular and noncardiovascular causes in the torcetrapib-treated group despite a $61 \%$ increase in HDL-cholesterol concentration and a $20 \%$ decrease in the level of LDL-cholesterol. ${ }^{19}$ Moreover, a multicenter, randomized, double-blind, placebo-controlled clinical trial, dal-OUTCOMES, was designed to test the hypothesis that dalcetrapib reduces cardiovascular morbidity and mortality in patients with recent ACS. However, dalcetrapib increased HDL-cholesterol levels, but did not reduce the risk of recurrent cardiovascular events. This trial was prematurely terminated because of a lack of benefit of dalcetrapib for patients with recent ACS..$^{25,30}$

\section{Reconstituted HDL and ApoA-I Mimetics}

HDL comprises heterogeneous particles with various densities 
and sizes, and ApoA-I is a common major protein of the HDL particle. ApoA-I-deficient and LDL receptor-deficient mice exhibit significant progression of atherosclerosis, approximately 5-fold increase than in LDL receptor-deficient mice, and this result demonstrates that ApoA-I deficiency is associated with a loss of protection from atherosclerotic development in a similar model of familial hypercholesterolemia. ${ }^{31} \mathrm{In}$ contrast, high levels of human ApoA-I lead to a significant increase in HDL-cholesterol levels and a decrease in the development of atherosclerotic plaque lesions in Apoe knockout mice. ${ }^{32,33}$

Numerous researchers are working to increase the quantity of cholesterol in the HDL particle and enhance the biochemical function of HDL as an approach to therapy. ${ }^{34,35}$ HDL-targeted therapies that inject full-length ApoA-I, reconstituted HDL (eg, the ApoA-I-phospholipid complex), or an ApoA-I mimetic peptide-lipid complex are remarkably effective (Figure 1). Reconstituted HDL must be disc-shaped and may be effective for treating patients with atherosclerosis.

ABCA1 plays a pivotal role in mediating phospholipid and cholesterol efflux of lipid-free ApoA-I as described earlier and is involved in the formation of the discoidal HDL precursor. Mature HDL particles, which are spherical, incorporate cellular cholesterol through sterol efflux via other $\mathrm{ABC}$ transporters such as ABCG1 and ABCG4. ${ }^{36}$ Rye et al prepared discoidal reconstituted HDL, which is complexed with human serum-derived ApoA-I containing 1-palmitoyl-2-oleoylphosphatidylcholine (POPC). ${ }^{37}$ The POPC/ApoA-I disc incorporates cholesterol derived from macrophages and is active in ABCA1-deficient Tangier disease patients (see later) as well as in normal subjects. ${ }^{38}$

ETC-642 is a form of reconstituted HDL with a predicted single amphipathic helix of 22 amino acid residues that mediates the formation of complexes of HDL with phospholipids. ${ }^{39}$ The characteristics of this reconstituted HDL are similar to those of the POPC/ApoA-I disc. The results of experiments using these reconstituted HDLs indicate that the ApoA-Iphospholipid complexes, but not the delipidated ApoA-I, mediate cholesterol efflux through ABCA1-dependent and nonspecific ABCA1-independent pathways. Thus, the main role of the ABCA1 transporter is to mediate the formation of the ApoA-I-phospholipid complex, and the artificial ApoA-Iphospholipid complex has the potential to incorporate cholesterol from cells via an ABCA1-independent pathway through, for example, the ABCG1 transporter. However, it is unclear whether ABCG1 and scavenger receptor class B member 1 mediate the efflux of cholesterol to reconstituted HDL independently or through the same pathway as the ApoA-I-phospholipid complex.

\section{ApoA-I Milano}

ApoA-I Milano is a mutant form of human ApoA-I that is generated by a point mutation that replaces an arginine with a cysteine residue at position 173. The levels of HDL-cholesterol and ApoA-I are markedly reduced in patients with this mutation. ${ }^{40,41}$ However, these patients are not at higher risk for cardiovascular disease. The higher levels of cellular cholesterol efflux mediated by ApoA-I Milano in cognate patients compared with subjects with ApoA-I are explained by the formation of ApoA-I Milano dimers linked by disulfide bonds between $\mathrm{C} 173$ residues. ${ }^{42,43}$

Chiesa et al conducted intravascular ultrasound (IVUS) and magnetic resonance imaging studies of rabbits fed a highcholesterol diet and found that ETC-216 [new code name: MDCO-216 (The Medicines Company)], an ApoA-I Milano-

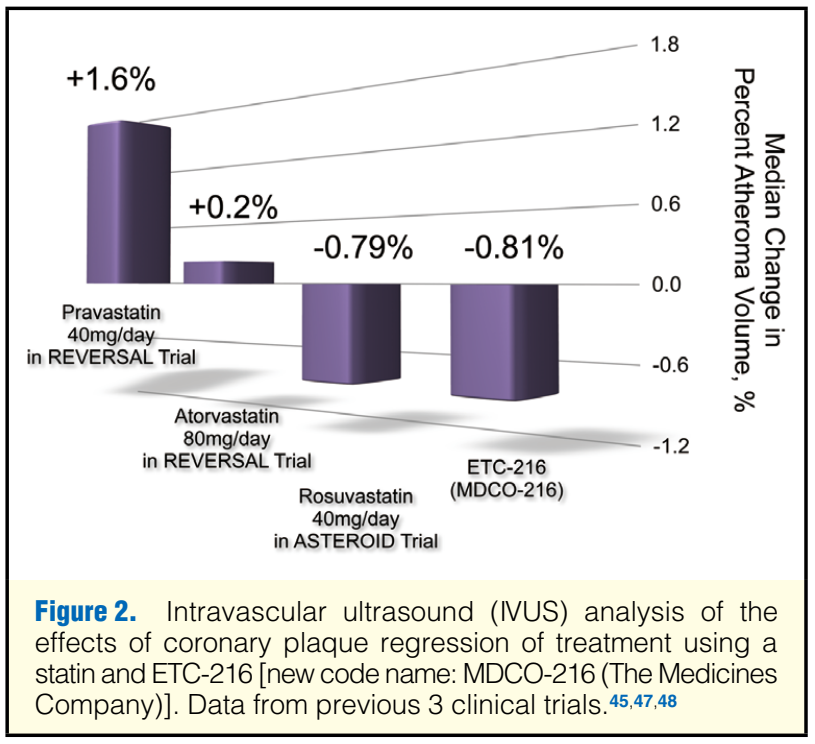

phospholipid complex (recombinant ApoA-I Milano complexed with POPC), significantly decreased the volume of carotid artery plaques. ${ }^{44}$ Nissen et al found that 5 weekly intravenous administrations of ETC-216 to humans lead to a regression of coronary atherosclerotic plaques. ${ }^{45}$ Furthermore, IVUS analysis revealed that after infusing ETC-216, regression of coronary atherosclerosis is accompanied by reverse remodeling of the external elastic membrane without changing luminal dimensions. ${ }^{46}$ Nissen et al conducted randomized, controlled clinical trials (REVERSAL ${ }^{47}$ and ASTEROID ${ }^{48}$ ) and used IVUS to investigate the effects of statins on the regression of coronary plaques. Comparison of the results of the 3 trials demonstrates that subacute ETC-216 treatment reduces coronary atheroma volume to the same extent as 18-24 months of intensive statin therapy (Figure 2).

\section{Fukuoka University APOA-I Mimetic Peptide (FAMP)}

The most severe form of HDL deficiency is Tangier disease, which was first described by Fredrickson et $\mathrm{al}^{49}$ and is caused by a mutation in $A B C A 1 .{ }^{50-52}$ Abcal knockout mice suffer from HDL deficiency and impaired RCT ${ }^{53,54}$ In contrast, ABCA1 overexpression increases ApoA-I-mediated cholesterol efflux in Abcal transgenic mice. ${ }^{55,56}$ These findings indicate that Abcal is pivotal for regulating plasma HDL-cholesterol levels as well as cellular cholesterol homeostasis.

Although studies of the use of ApoA-I mimetic peptides, such as D-4F, L-4F (APL180), and L37pA, are underway, ${ }^{57-60}$ none of these agents are currently available for clinical use. Moreover, these peptides have a high affinity for lipids, so they appear to increase cholesterol efflux through a nonspecific, passive pathway. The recently described novel human ApoA-I-mimetic 24-mer peptide [FAMP, one-tenth of human ApoA-I in molecular weight], which does not form complexes with phospholipids, ${ }^{61}$ significantly enhances the biochemical function of HDL and reduces the formation of aortic plaques by $48.2 \%$ in Apoe knockout mice fed a high-fat diet. ${ }^{62}$ FAMP differs from other ApoA-I mimetics because it is designed to specifically interact with human ABCA1 without engaging the nonspecific, passive efflux pathway. Therefore, it functions similarly to human ApoA-I. Furthermore, FAMP markedly increases pre- $\beta$ HDL as well as overall cholesterol efflux from peripheral tissues. 


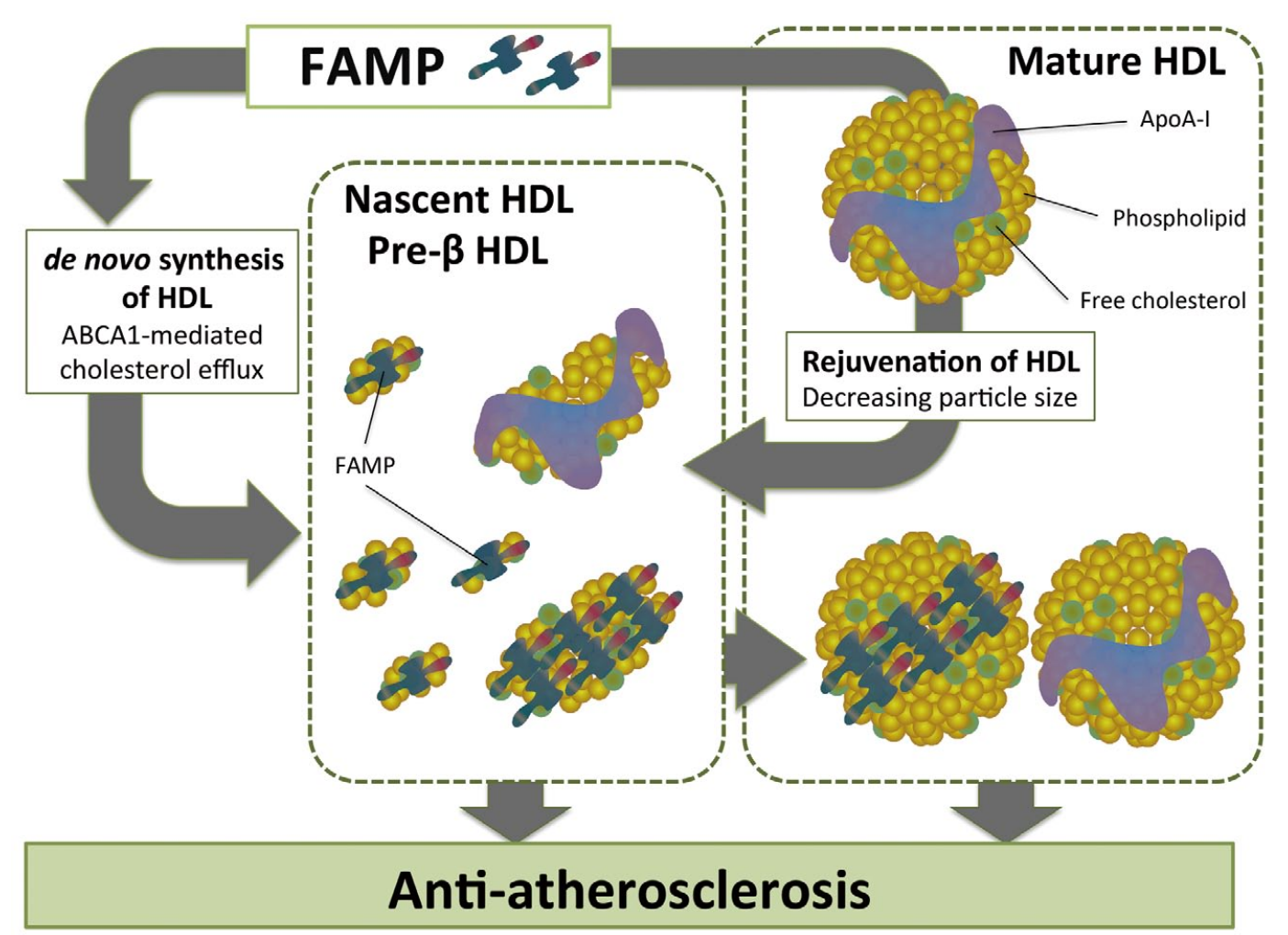

Figure 3. A possibly distinct therapeutic role of FAMP that modifies HDL metabolism. FAMP plays at least 2 distinct roles in HDL metabolism. First, it enhances cellular cholesterol efflux that is mediated through an ABCA1-dependent pathway, de novo generating nascent pre- $\beta$ HDL particles. Second, FAMP incubation with human HDL or plasma generates pre- $\beta$-HDL-like small HDL particles and charged ApoA-I-rich particles that accelerate the generation of pre- $\beta \mathrm{HDL}$, which is derived from mature HDL. ABCA1, ATP-binding cassette A1; Apo, apolipoprotein; FAMP, Fukuoka University APOA-I mimetic peptide; HDL, high-density lipoprotein.

FAMP plays at least 2 possibly distinct roles in HDL metabolism as follows. First, FAMP enhances cellular cholesterol efflux that is mediated through ABCA1-dependent and partly ABCA1-independent mechanisms, generating nascent pre- $\beta$ HDL particles. Second, FAMP incubation with human HDL or plasma generates pre- $\beta$-HDL-like small HDL particles and charged ApoA-I-rich particles that accelerate the generation of pre- $\beta$ HDL, which is derived from mature HDL (Figure 3). Although 16-week treatment of Apoe knockout mice with FAMP did not significantly increase the level of HDL-cholesterol, the free cholesterol levels of the small-size HDL subfractions, reflecting pre- $\beta_{1}$ HDL particles, were significantly increased, and this should reflect enhanced de novo generation of HDL through cholesterol efflux from peripheral tissue in the treated mice. On the one hand, CETP inhibition greatly increasing HDL-cholesterol levels is attended to grow larger HDL particle size; on the other hand, the apoA-I mimetic peptide FAMP reduces the particle size without changing the HDL-cholesterol level; thus these phenomena seem to be precisely opposing.

Moreover, the biological function of HDL in FAMP-treated Apoe knockout mice is significantly increased, according to the results of an analysis of ex vivo HDL efflux capacity that is strongly predictive of coronary heart disease. ${ }^{63}$ Furthermore, FAMP promotes ABCA1-dependent HDL efflux ex vivo, HDL turnover in vivo, and RCT in the macrophages of transgenic mice that express human CETP, despite decreased plasma HDL-cholesterol levels. ${ }^{64}$ These findings support the conclusion that the prospects are promising for successful treatment of atherosclerotic disease with FAMP and other mimetics.

HDL may play a significant role in the pathogenesis of diseases other than those of the cardiovascular system. For example, mice with complete HDL deficiency caused by targeted deletions of Apoal and Apoe exhibit a phenotype characterized by deep alterations in skin structure, with a massive dermal accumulation of cholesterol clefts, foam cells, and $\mathrm{T}$ lymphocytes. ${ }^{65}$ This phenotype resembles that of humans with inherited or secondary hyperlipidemic conditions referred to as xanthomas or xanthelasmas. Therefore, HDL-targeted therapy may be applicable to patients with xanthomas, as well as to those with dermatitis.

Besides its therapeutic potential, FAMP exhibits interesting properties that can be applied to the analysis of atherosclerotic plaque. ApoA-I or its mimetics must penetrate atherosclerotic plaques to remove cholesterol, and this characteristic may be exploited to detect activated arterial plaques. FAMP serves as a unique tracer for positron electron emission tomography (PET). When FAMP is functionalized using the chelator, 1,4,7,10-tetraazacyclododecane-1,4,7,10-tetraacetic acid and labeled with ${ }^{68} \mathrm{Ga}\left({ }^{68} \mathrm{Ga}-\mathrm{DOTA}-\mathrm{FAMP}\right)$, it can be used to 
specifically image atherosclerotic plaques. Thus, atherosclerotic plaques incorporate ${ }^{68} \mathrm{Ga}$-DOTA-FAMP at a high rate, generating impressive PET images of an aortic plaque in vivo. ${ }^{66}$ Uchida et al have also investigated plaque characteristics using color fluorescent microscopy and shown the fluorescent characteristics of HDL deposits with plaque formation, but not in the advanced stage of plaque in the human coronary arterial wall. ${ }^{67}$

We believe that HDL-targeted therapy, including the use of FAMP, has tremendous atheroprotective potential and likely represents a new therapeutic tool for treating atherosclerotic cardiovascular disease. Although most research is focused on the therapeutic use of HDL, an ApoA-I mimetic peptide may contribute to the development of a technique to diagnose lipidrich, unstable plaques.

\section{Conclusions}

LDL-lowering statin therapy is the standard treatment for cardiovascular diseases; however, it lacks benefit for preventing or mitigating adverse vascular events experienced by numerous patients. Therefore, the studies described here indicate that targeting HDL has very bright prospects for treating patients with atherosclerotic diseases. In particular, novel ApoA-I mimetics, such as FAMP, will likely enhance the pharmacological armamentarium available for treating these diseases.

\section{Acknowledgments}

This work was supported by Grants-in-Aid for Scientific Research (No. 19590874, No. 25461141 and No. 21590960) from the Ministry of Education, Culture, Sports, Science, and Technology of Japan to Y.U. and K.S.; and in part by funds from the Central Research Institute of Fukuoka University (2013-2015) to Y.U. and K.S., the Fukuoka University OneCampus Project (2009, 2010, supported in part by the Ministry of Education, Science and Culture of Japan), supported in part by funds (No. 157009) from the Central Research Institute of Fukuoka University to Y.U. and NPO Clinical and Applied Science, Fukuoka, Japan.

\section{Disclosures}

Disclosures (Y.U., G.C., and K.S.): None.

K.S. is the Chief Director of NPO Clinical and Applied Science, Fukuoka, Japan.

\section{References}

1. von Eckardstein A, Nofer JR, Assmann G. High density lipoproteins and arteriosclerosis: Role of cholesterol efflux and reverse cholesterol transport. Arterioscler Thromb Vasc Biol 2001; 21: 13-27.

2. Gordon T, Castelli WP, Hjortland MC, Kannel WB, Dawber TR. High density lipoprotein as a protective factor against coronary heart disease: The Framingham study. Am J Med 1977; 62: 707-714.

3. Assmann G. Genes and dyslipoproteinaemias. Eur Heart J 1990; 11(Suppl H): 4-8.

4. Briel M, Ferreira-Gonzalez I, You JJ, Karanicolas PJ, Akl EA, Wu $\mathrm{P}$, et al. Association between change in high density lipoprotein cholesterol and cardiovascular disease morbidity and mortality: Systematic review and meta-regression analysis. BMJ 2009; 338: b92.

5. Saku K, Zhang B, Noda K, Investigators PT. Randomized head-tohead comparison of pitavastatin, atorvastatin, and rosuvastatin for safety and efficacy (quantity and quality of LDL): The PATROL trial. Circ J 2011; 75: 1493-1505.

6. Soeda T, Uemura S, Okayama S, Kawakami R, Sugawara Y, Nakagawa $\mathrm{H}$, et al. Intensive lipid-lowering therapy with rosuvastatin stabilizes lipid-rich coronary plaques: Evaluation using dualsource computed tomography. Circ J 2011; 75: 2621-2627.

7. Havel RJ, Eder HA, Bragdon JH. The distribution and chemical composition of ultracentrifugally separated lipoproteins in human serum. J Clin Invest 1955; 34: 1345-1353.

8. Kunitake ST, La Sala KJ, Kane JP. Apolipoprotein A-I-containing lipoproteins with pre-beta electrophoretic mobility. J Lipid Res 1985; 26: $549-555$.
9. Ishida BY, Frolich J, Fielding CJ. Prebeta-migrating high density lipoprotein: Quantitation in normal and hyperlipidemic plasma by solid phase radioimmunoassay following electrophoretic transfer. $J$ Lipid Res 1987; 28: 778-786.

10. Miller M, Rhyne J, Hamlette S, Birnbaum J, Rodriguez A. Genetics of HDL regulation in humans. Curr Opin Lipidol 2003; 14: $273-$ 279.

11. Oram JF, Lawn RM. ABCA1: The gatekeeper for eliminating excess tissue cholesterol. J Lipid Res 2001; 42: 1173-1179.

12. Barter PJ, Brewer HB Jr, Chapman MJ, Hennekens CH, Rader DJ, Tall AR. Cholesteryl ester transfer protein: A novel target for raising HDL and inhibiting atherosclerosis. Arterioscler Thromb Vasc Biol 2003; 23: 160-167.

13. Yamashita S, Sprecher DL, Sakai N, Matsuzawa Y, Tarui S, Hui DY. Accumulation of apolipoprotein E-rich high density lipoproteins in hyperalphalipoproteinemic human subjects with plasma cholesteryl ester transfer protein deficiency. J Clin Invest 1990; 86: 688-695.

14. Clark RW, Sutfin TA, Ruggeri RB, Willauer AT, Sugarman ED, Magnus-Aryitey G, et al. Raising high-density lipoprotein in humans through inhibition of cholesteryl ester transfer protein: An initial multidose study of torcetrapib. Arterioscler Thromb Vasc Biol 2004; 24: 490-497.

15. Brousseau ME, Schaefer EJ, Wolfe ML, Bloedon LT, Digenio AG, Clark RW, et al. Effects of an inhibitor of cholesteryl ester transfer protein on HDL cholesterol. N Engl J Med 2004; 350: 1505-1515.

16. Ikewaki K, Rader DJ, Sakamoto T, Nishiwaki M, Wakimoto N, Schaefer JR, et al. Delayed catabolism of high density lipoprotein apolipoproteins A-I and A-II in human cholesteryl ester transfer protein deficiency. J Clin Invest 1993; 92: 1650-1658.

17. Tall AR. Plasma high density lipoproteins: Metabolism and relationship to atherogenesis. J Clin Invest 1990; 86: 379-384.

18. Cuchel M, Rader DJ. Genetics of increased HDL cholesterol levels: Insights into the relationship between HDL metabolism and atherosclerosis. Arterioscler Thromb Vasc Biol 2003; 23: 1710-1712.

19. Nicholls SJ, Tuzcu EM, Brennan DM, Tardif JC, Nissen SE. Cholesteryl ester transfer protein inhibition, high-density lipoprotein raising, and progression of coronary atherosclerosis: Insights from ILLUSTRATE (investigation of lipid level management using coronary ultrasound to assess reduction of atherosclerosis by CETP inhibition and HDL elevation). Circulation 2008; 118: 2506-2514.

20. Bots ML, Visseren FL, Evans GW, Riley WA, Revkin JH, Tegeler $\mathrm{CH}$, et al. Torcetrapib and carotid intima-media thickness in mixed dyslipidaemia (Radiance 2 study): A randomised, double-blind trial. Lancet 2007; 370: 153-160.

21. Kastelein JJ, van Leuven SI, Burgess L, Evans GW, Kuivenhoven $\mathrm{JA}$, Barter PJ, et al. Effect of torcetrapib on carotid atherosclerosis in familial hypercholesterolemia. N Engl J Med 2007; 356: 1620-1630.

22. Nissen SE, Tardif JC, Nicholls SJ, Revkin JH, Shear CL, Duggan WT, et al. Effect of torcetrapib on the progression of coronary atherosclerosis. N Engl J Med 2007; 356: 1304-1316.

23. Fayad ZA, Mani V, Woodward M, Kallend D, Abt M, Burgess T, et al. Safety and efficacy of dalcetrapib on atherosclerotic disease using novel non-invasive multimodality imaging (dal-PLAQUE): A randomised clinical trial. Lancet 2011; 378: 1547-1559.

24. Luscher TF, Taddei S, Kaski JC, Jukema JW, Kallend D, Munzel T, et al. Vascular effects and safety of dalcetrapib in patients with or at risk of coronary heart disease: The dal-VESSEL randomized clinical trial. Eur Heart J 2012; 33: 857-865.

25. Schwartz GG, Olsson AG, Abt M, Ballantyne CM, Barter PJ, Brumm J, et al. Effects of dalcetrapib in patients with a recent acute coronary syndrome. N Engl J Med 2012; 367: 2089-2099.

26. Cannon CP, Braunwald E, McCabe CH, Rader DJ, Rouleau JL, Belder R, et al. Intensive versus moderate lipid lowering with statins after acute coronary syndromes. N Engl J Med 2004; 350: $1495-$ 1504.

27. Bloomfield D, Carlson GL, Sapre A, Tribble D, McKenney JM, Littlejohn TW 3rd, et al. Efficacy and safety of the cholesteryl ester transfer protein inhibitor anacetrapib as monotherapy and coadministered with atorvastatin in dyslipidemic patients. Am Heart J 2009; 157: 352-360.e2, doi:10.1016/j.ahj.2008.09.022.

28. Nicholls SJ, Brewer HB, Kastelein JJ, Krueger KA, Wang MD, Shao $\mathrm{M}$, et al. Effects of the CETP inhibitor evacetrapib administered as monotherapy or in combination with statins on HDL and LDL cholesterol: Arandomized controlled trial. JAMA 2011; 306: 20992109.

29. Cao G, Beyer TP, Zhang Y, Schmidt RJ, Chen YQ, Cockerham SL, et al. Evacetrapib is a novel, potent, and selective inhibitor of cholesteryl ester transfer protein that elevates HDL cholesterol without inducing aldosterone or increasing blood pressure. J Lipid Res 2011; 52: $2169-2176$. 
30. Schwartz GG, Olsson AG, Ballantyne CM, Barter PJ, Holme IM, Kallend D, et al. Rationale and design of the dal-OUTCOMES trial: Efficacy and safety of dalcetrapib in patients with recent acute coronary syndrome. Am Heart J 2009; 158: 896-901.e3, doi:10.1016/j. ahj.2009.09.017.

31. Moore RE, Kawashiri MA, Kitajima K, Secreto A, Millar JS, Pratico $\mathrm{D}$, et al. Apolipoprotein A-I deficiency results in markedly increased atherosclerosis in mice lacking the LDL receptor. Arterioscler Thromb Vasc Biol 2003; 23: 1914-1920.

32. Paszty C, Maeda N, Verstuyft J, Rubin EM. Apolipoprotein AI transgene corrects apolipoprotein E deficiency-induced atherosclerosis in mice. J Clin Invest 1994; 94: 899-903.

33. Plump AS, Scott CJ, Breslow JL. Human apolipoprotein A-I gene expression increases high density lipoprotein and suppresses atherosclerosis in the apolipoprotein E-deficient mouse. Proc Natl Acad Sci USA 1994; 91: 9607-9611

34. Annema W, von Eckardstein A. High-density lipoproteins: Multifunctional but vulnerable protections from atherosclerosis. Circ J 2013; 77: 2432-2448.

35. Zhang B, Kawachi E, Miura S, Uehara Y, Matsunaga A, Kuroki M, et al. Therapeutic approaches to the regulation of metabolism of high-density lipoprotein: Novel HDL-directed pharmacological intervention and exercise. Circ J 2013; 77: 2651-2663.

36. Wang N, Lan D, Chen W, Matsuura F, Tall AR. ATP-binding cassette transporters G1 and G4 mediate cellular cholesterol efflux to high-density lipoproteins. Proc Natl Acad Sci USA 2004; 101: 9774-9779.

37. Rye KA, Hime NJ, Barter PJ. Evidence that cholesteryl ester transfer protein-mediated reductions in reconstituted high density lipoprotein size involve particle fusion. J Biol Chem 1997; 272: 3953-3960.

38. Uehara Y, Tsuboi Y, Zhang B, Miura S, Baba Y, Higuchi MA, et al. POPC/ApoA-I discs as a potent lipoprotein modulator in Tangier disease. Atherosclerosis 2008; 197: 283-289.

39. Iwata A, Miura S, Zhang B, Imaizumi S, Uehara Y, Shiomi M, et al. Antiatherogenic effects of newly developed apolipoprotein A-I mimetic peptide/phospholipid complexes against aortic plaque burden in Watanabe-heritable hyperlipidemic rabbits. Atherosclerosis 2011; 218: 300-307.

40. Weisgraber KH, Bersot TP, Mahley RW, Franceschini G, Sirtori CR. A-IMilano apoprotein: Isolation and characterization of a cysteinecontaining variant of the A-I apoprotein from human high density lipoproteins. J Clin Invest 1980; 66: 901-907.

41. Gualandri V, Franceschini G, Sirtori CR, Gianfranceschi G, Orsini $\mathrm{GB}$, Cerrone A, et al. AIMilano apoprotein identification of the complete kindred and evidence of a dominant genetic transmission. Am J Hum Genet 1985; 37: 1083-1097.

42. Favari E, Gomaraschi M, Zanotti I, Bernini F, Lee-Rueckert M, Kovanen PT, et al. A unique protease-sensitive high density lipoprotein particle containing the apolipoprotein A-I(Milano) dimer effectively promotes ATP-binding cassette A1-mediated cell cholesterol efflux. J Biol Chem 2007; 282: 5125-5132.

43. Franceschini G, Calabresi L, Chiesa G, Parolini C, Sirtori CR, Canavesi M, et al. Increased cholesterol efflux potential of sera from apoA-IMilano carriers and transgenic mice. Arterioscler Thromb Vasc Biol 1999; 19: 1257-1262.

44. Parolini C, Marchesi M, Lorenzon P, Castano M, Balconi E, Miragoli L, et al. Dose-related effects of repeated ETC-216 (recombinant apolipoprotein A-I Milano/1-palmitoyl-2-oleoyl phosphatidylcholine complexes) administrations on rabbit lipid-rich soft plaques: In vivo assessment by intravascular ultrasound and magnetic resonance imaging. J Am Coll Cardiol 2008; 51: 1098-1103.

45. Nissen SE, Tsunoda T, Tuzcu EM, Schoenhagen P, Cooper CJ, Yasin M, et al. Effect of recombinant apoA-I Milano on coronary atherosclerosis in patients with acute coronary syndromes: A randomized controlled trial. JAMA 2003; 290: 2292-2300.

46. Nicholls SJ, Tuzcu EM, Sipahi I, Schoenhagen P, Crowe T, Kapadia $\mathrm{S}$, et al. Relationship between atheroma regression and change in lumen size after infusion of apolipoprotein A-I Milano. J Am Coll Cardiol 2006; 47: 992-997.

47. Nissen SE, Tuzcu EM, Schoenhagen P, Brown BG, Ganz P, Vogel RA, et al. Effect of intensive compared with moderate lipid-lowering therapy on progression of coronary atherosclerosis: A randomized controlled trial. JAMA 2004; 291: 1071-1080.

48. Nissen SE, Nicholls SJ, Sipahi I, Libby P, Raichlen JS, Ballantyne $\mathrm{CM}$, et al. Effect of very high-intensity statin therapy on regression of coronary atherosclerosis: The ASTEROID trial. JAMA 2006; 295: $1556-1565$.

49. Fredrickson DS, Altrocchi PH, Avioli LV, Goodman DS, Goodman HC. Tangier disease: Combined clinical staff conference at the National Institutes of Health. Ann Intern Med 1961; 55: 1016-1031.

50. Brooks-Wilson A, Marcil M, Clee SM, Zhang LH, Roomp K, van Dam M, et al. Mutations in ABC1 in Tangier disease and familial high-density lipoprotein deficiency. Nat Genet 1999; 22: 336-345.

51. Bodzioch M, Orso E, Klucken J, Langmann T, Bottcher A, Diederich $\mathrm{W}$, et al. The gene encoding ATP-binding cassette transporter 1 is mutated in Tangier disease. Nat Genet 1999; 22: 347-351.

52. Rust S, Rosier M, Funke H, Real J, Amoura Z, Piette JC, et al. Tangier disease is caused by mutations in the gene encoding ATPbinding cassette transporter 1. Nat Genet 1999; 22: 352-355.

53. Orso E, Broccardo C, Kaminski WE, Bottcher A, Liebisch G, Drobnik W, et al. Transport of lipids from Golgi to plasma membrane is defective in Tangier disease patients and $\mathrm{ABC} 1$-deficient mice. Nat Genet 2000; 24: $192-196$.

54. McNeish J, Aiello RJ, Guyot D, Turi T, Gabel C, Aldinger C, et al. High density lipoprotein deficiency and foam cell accumulation in mice with targeted disruption of ATP-binding cassette transporter-1. Proc Natl Acad Sci USA 2000; 97: 4245-4250.

55. Vaisman BL, Lambert G, Amar M, Joyce C, Ito T, Shamburek RD, et al. ABCA1 overexpression leads to hyperalphalipoproteinemia and increased biliary cholesterol excretion in transgenic mice. J Clin Invest 2001; 108: $303-309$.

56. Singaraja RR, Bocher V, James ER, Clee SM, Zhang LH, Leavitt $\mathrm{BR}$, et al. Human ABCA1 BAC transgenic mice show increased high density lipoprotein cholesterol and apoAI-dependent efflux stimulated by an internal promoter containing liver $\mathrm{X}$ receptor response elements in intron 1. J Biol Chem 2001; 276: 33969-33979.

57. Navab M, Anantharamaiah GM, Hama S, Garber DW, Chaddha M, Hough G, et al. Oral administration of an apo A-I mimetic peptide synthesized from D-amino acids dramatically reduces atherosclerosis in mice independent of plasma cholesterol. Circulation 2002; 105: 290-292.

58. Li X, Chyu KY, Faria Neto JR, Yano J, Nathwani N, Ferreira C, et al. Differential effects of apolipoprotein A-I-mimetic peptide on evolving and established atherosclerosis in apolipoprotein E-null mice. Circulation 2004; 110: 1701-1705.

59. Remaley AT, Thomas F, Stonik JA, Demosky SJ, Bark SE, Neufeld $\mathrm{EB}$, et al. Synthetic amphipathic helical peptides promote lipid efflux from cells by an ABCA1-dependent and an ABCA1-independent pathway. J Lipid Res 2003; 44: 828-836.

60. Van Lenten BJ, Wagner AC, Jung CL, Ruchala P, Waring AJ, Lehrer RI, et al. Anti-inflammatory apoA-I-mimetic peptides bind oxidized lipids with much higher affinity than human apoA-I. J Lipid Res 2008; 49: 2302-2311.

61. Uehara Y, Ando S, Oniki K, Abe S, Yahiro E, Tanigawa H, et al. FAMP, a novel apoA-I mimetic peptide promotes HDL via ABCA1dependent cholesterol efflux. Atherosclerosis Suppl 2010; 11: 3 .

62. Uehara Y, Ando S, Yahiro E, Oniki K, Ayaori M, Abe S, et al. FAMP, a novel apoA-I mimetic peptide, suppresses aortic plaque formation through promotion of biological HDL function in apoEdeficient mice. J Am Heart Assoc 2013; 2: e000048, doi:10.1161/ JAHA.113.000048.

63. Khera AV, Cuchel M, de la Llera-Moya M, Rodrigues A, Burke MF, Jafri K, et al. Cholesterol efflux capacity, high-density lipoprotein function, and atherosclerosis. N Engl J Med 2011; 364: 127-135.

64. Shimizu T, Tanigawa H, Miura S, Kuwano T, Takata K, Suematsu Y, et al. Newly developed apolipoprotein A-I mimetic peptide promotes macrophage reverse cholesterol transport in vivo. Int J Cardiol 2015; 192: $82-88$.

65. Arnaboldi F, Busnelli M, Cornaghi L, Manzini S, Parolini C, Dellera F, et al. High-density lipoprotein deficiency in genetically modified mice deeply affects skin morphology: A structural and ultrastructural study. Exp Cell Res 2015; 338: 105-112.

66. Kawachi E, Uehara Y, Hasegawa K, Yahiro E, Ando S, Wada Y, et al. Novel molecular imaging of atherosclerosis with gallium-68-labeled apolipoprotein A-I mimetic peptide and positron emission tomography. Circ J 2013; 77: 1482-1489.

67. Uchida Y, Hiruta N, Yamanoi D, Shimoyama E, Maezawa Y, Uchida Y. Imaging of native high-density lipoprotein in human coronary plaques by color fluorescent angioscopy. Circ J 2014; 78: $1667-1675$. 